\title{
Efecto del mínimo procesamiento y tratamiento con yerba mate sobre el potencial saludable y los atributos de calidad de manzanas Granny Smith
}

\section{Effect of minimal processing and treatment with yerba mate on healthy potential and quality attributes of Granny Smith apples}

Rodríguez Arzuaga, Mariana (1-2), Salsi, María Sara (2), Piagentini, Andrea Marcela (2)

(1) Departamento de Análisis de productos agropecuarios, Laboratorio Tecnológico del Uruguay, LATU

(2) Instituto de Tecnología de Alimentos, Facultad de Ingeniería Química, Universidad Nacional del Litoral, Santa Fe, Argentina.

Contacto: marodrig@latu.org.uy

RECIBIDO: $12 / 8 / 2016$ - APROBADO: $25 / 11 / 2016$

\begin{abstract}
Resumen
Se estudió el efecto del mínimo procesamiento solo y con tratamiento con ácidos cítrico y ascórbico e infusión de yerba mate, sobre las características fisicoquímicas, bioactivas, microbiológicas y sensoriales de manzanas Granny Smith. El mínimo procesamiento (sin tratamiento químico) produjo un descenso en el contenido de sólidos solubles y compuestos polifenólicos, en el recuento de aerobios mesófilos totales y en los atributos sensoriales gusto ácido y pardeamiento, y una mejora en la apariencia general evaluada sensorialmente. Comparando los resultados obtenidos para las manzanas mínimamente procesadas tratadas químicamente y sin tratar (control), se observó que el tratamiento químico aplicado produjo una disminución del sabor característico de las manzanas y un incremento en el gusto ácido y olores y sabores extraños. Sin embargo, el tratamiento aplicado fue efectivo en controlar el desarrollo de pardeamiento (aumento de $\mathrm{L}^{*} \mathrm{y}$ mejora de la apariencia general sensorial y disminución de $\mathrm{a}^{\star}$ y del pardeamiento evaluado sensorialmente) y en aumentar el potencial saludable (mayor contenido de polifenoles y vitamina $\mathrm{C}$ y mayor capacidad antioxidante), manteniendo recuentos microbiológicos similares a los encontrados en la materia prima. Palabras clave: Frutas frescas cortadas, capacidad antioxidante, color.
\end{abstract}

\begin{abstract}
The effect of the minimal processing with and without treatment with citric and ascorbic acids and yerba mate on the physicochemical, bioactive, microbiological and sensory characteristics of Granny Smith apples was studied. Soluble solids and polyphenol contents, total aerobic mesophilic microorganism counts, sour taste and the browning sensory attribute were reduced by the minimal processing (without chemical treatment), while the sensory general appearance improved. When comparing the results obtained for the treated and untreated (control) freshcut apples, it was observed that the applied chemical treatment reduced the characteristic flavor of the apples and increased sour taste, off-flavors and off-odors. However, the treatment was effective in controlling the browning development ( $\mathrm{L}^{*}$ increased, sensory general appearance improved and $\mathrm{a}^{*}$ and sensory attribute browning decreased), in increasing the healthy potential (higher polyphenol and vitamin C content and antioxidant capacity), and maintaining the microbial counts similar to those obtained for raw material.

Keywords: Fresh-cut fruits, antioxidant capacity, color.
\end{abstract}

\section{Introducción}

Las frutas y hortalizas frescas son componentes esenciales de la dieta humana y existe evidencia considerable de los beneficios nutricionales y para la salud asociados a su consumo, debido a su contenido en vitaminas, minerales, fibras y compuestos biológicamente activos (Cano, et al., 2005; Alegre, et al., 2011). La introducción en el mercado de frutas y hortalizas mínimamente procesadas es una herramienta de gran importancia para facilitar la incorporación de estos alimentos en la dieta del consumidor.
Cada una de las etapas de elaboración de frutas y hortalizas frescas cortadas puede influir en su calidad microbiológica (Piagentini, et al., 2003; Pirovani, et al., 2004), atributos sensoriales (Piagentini, et al., 2002; Pirovani, et al., 2009), potencial saludable (Pirovani, et al., 2015) y, por lo tanto, en su vida útil (Piagentini y Güemes, 2002). La vida útil de los productos frescos cortados es inferior a la de las frutas y hortalizas intactas de las que provienen, debido a que durante la elaboración sufren daño en su tejido, princi- 
palmente en las etapas de pelado y corte, incrementando el deterioro microbiológico e induciendo cambios fisiológicos y bioquímicos que generan degradación del color, la textura y el sabor (Chung y Moon, 2009).

El pardeamiento enzimático imparte características sensoriales que desestimulan al consumidor a comprar frutas mínimamente procesadas y es considerado una de las principales limitantes de la vida útil de manzanas frescas cortadas (Rico, et al., 2007). Se han propuesto numerosas alternativas para solucionar este problema, entre las que se destacan los tratamientos con soluciones antioxidantes, que son una de las barreras utilizadas para preservar el color inicial en frutas frescas cortadas. El ácido ascórbico y sus derivados (solo o en combinación con ácido cítrico y/o sales de calcio) se han usado en varios estudios en frutas y hortalizas y su efecto para prevenir el pardeamiento ha sido demostrado (Piagentini y Güemes, 2002; Toivonen y Brummell, 2008; Piagentini, et al., 2012; Rodríguez Arzuaga, et al., 2013a). Sin embargo, la mayoría de estos agentes químicos tienen eficiencia variable y pueden generar sabores extraños.

El interés en la utilización de compuestos naturales para prevenir la pérdida de calidad en frutas frescas cortadas se ha incrementado notablemente como consecuencia del deseo del consumidor de reducir o eliminar los aditivos sintetizados químicamente en los alimentos (Lanciotti, et al., 2004). La yerba mate se elabora a partir de las hojas maduras de Ilex paraguariensis que son cosechadas, desecadas y luego molidas. Algunas de las propiedades farmacológicas atribuidas al mate se han relacionado con su alto contenido en compuestos fenólicos. El alto contenido de compuestos polifenólicos en el mate es responsable de sus notables propiedades antioxidantes (Bravo, et al., 2007) y lo convierten en un excelente candidato para aplicar como antioxidante natural a frutas frescas cortadas. Martín et al. (2010) encontraron que infusiones de yerba mate aplicadas a extractos de la enzima polifenol oxidasa (PPO) de manzanas ejercieron un significativo efecto inhibitorio sobre la actividad de dicha enzima. A pesar de su potencial efecto beneficioso, la investigación sobre la aplicación de soluciones de yerba mate en frutas frescas cortadas es sumamente limitada.

Por lo tanto, el objetivo de este trabajo fue estudiar el efecto del mínimo procesamiento y de la aplicación de un tratamiento químico con una solución compuesta por $0,9 \%$ de ácido cítrico (ÁC), 1,0\% de ácido ascórbico (ÁA) y 1,2\% de yerba mate (YM) sobre las características fisicoquímicas, bioactivas, microbiológicas y sensoriales de manzanas Granny Smith.

\section{Materiales y Métodos}

\section{Preparación de las muestras}

\section{Materia Prima (MP)}

Se trabajó con manzanas variedad Granny Smith adquiridas en un comercio local de la ciudad de Santa Fe, Argentina. Teniendo en cuenta su menor susceptibilidad al desarrollo de pardeamiento y mayor firmeza y jugosidad, esta variedad es, entre las disponibles comercialmente, una de las más adecuadas para el procesamiento mínimo (Piagentini y
Pirovani, 2016). Una vez recibida la materia prima (20 kg), se almacenó en cámara refrigerada a $1,5^{\circ} \mathrm{C}$ hasta el momento de la preparación.

\section{Preparación de la infusión de yerba mate (YM)}

Se utilizó YM comercial de origen argentino del mismo lote, adquirida en un comercio de la zona. YM previamente pesada se agregó a agua a $90^{\circ} \mathrm{C}(1,2$ g por cada $100 \mathrm{~mL})$, se agitó y se mantuvo tapada y en reposo durante 5 minutos. Luego se filtró con algodón, se dejó alcanzar temperatura ambiente y se agregó agua hasta completar el volumen total inicial (para compensar las pérdidas por evaporación). La infusión fue nuevamente filtrada utilizando papel de filtro.

\section{Muestras control (C) y tratadas (T)}

Las manzanas se lavaron con $100 \mathrm{ppm} \mathrm{de} \mathrm{Cl}_{2}\left(\mathrm{pH} 7,0 ; 20^{\circ} \mathrm{C}\right)$, se pelaron, descorazonaron y cortaron en octavos. Los octavos se desinfectaron con $30 \mathrm{ppm} \mathrm{de} \mathrm{Cl}_{2}\left(\mathrm{pH} \mathrm{7,0;20}{ }^{\circ} \mathrm{C}\right)$. Las muestras $\mathrm{C}$ se sumergieron en agua y las $\mathrm{T}$ en una solución $\operatorname{de}[1,2 \% \mathrm{YM}+0,9 \%$ ÁC + 1,0\% ÂA] (concentraciones determinadas previamente por Rodríguez Arzuaga, et al., 2013b) durante 3 minutos en una relación de $1 \mathrm{~kg}$ manzana por $3 \mathrm{~L}$ de solución. Se escurrieron por gravedad y por último sobre papel absorbente. Los octavos de manzana correspondientes a ambas muestras (C y T) se envasaron en bandejas de tereftlato de polietileno (PET) con tapa y se almacenaron en cámara a $1,5^{\circ} \mathrm{C}$ hasta el momento de su análisis. Todos los ensayos se realizaron en el día del procesamiento de las manzanas.

\section{Análisis fisicoquímicos}

$$
\text { pH y sólidos solubles }
$$

El pH se midió con un pH-metro Horiba B-213 y los sólidos solubles con un refractómetro digital "Pocket" ATAGO PAL-ALFA. Los resultados fueron expresados en ${ }^{\circ}$ Brix.

\section{Firmeza}

Se determinó sobre la pulpa de la fruta utilizando un penetrómetro Penefel DFT Digital Firmness tester (Agro Technologies) con una punta de $11 \mathrm{~mm}$. Los resultados se expresaron en Newton (N).

\section{Color}

Se determinó sobre la superficie cortada de la fruta con un espectrofotómetro Minolta 508d, iluminante D65, $10^{\circ}$ y SCE, midiendo los parámetros CIELAB: $\mathrm{L}^{*}, \mathrm{a}^{*} \mathrm{y} \mathrm{b}^{*}$. También se determinaron los parámetros $\mathrm{C}_{\mathrm{ab}}{ }^{*}=\left(\mathrm{a}^{\star 2}+\mathrm{b}^{\star 2}\right)^{0,5} \mathrm{y}$ el ángulo de tono $\mathrm{h}_{\mathrm{ab}}=\arctan \left(\mathrm{b}^{\star} / \mathrm{a}^{\star}\right)$.

\section{Polifenoles y capacidad antioxidante}

\section{Extracción}

Se agregó acetona/agua 80/20 a las muestras molidas en una relación peso muestra:volumen solvente de 1:10. Luego se llevaron al ultrasonido por 15 minutos y se separó el sobrenadante 
por centrifugación para la determinación de polifenoles totales y capacidad antioxidante (Rodríguez Arzuaga, et al., 2013a).

\section{Polifenoles totales}

Se determinaron de acuerdo al método de Folin-Ciocalteu modificado por Singleton y Rossi (1965) a través de la medida de la absorbancia a $760 \mathrm{~nm}$, utilizando un espectrofotómetro UV-Visible - MILTON ROY - Spectronic Génesis 5. Los resultados se expresaron en miligramos de ácido gálico equivalente (ÁGE) por $100 \mathrm{~g}$.

\section{Capacidad antioxidante por el método del radical $\mathrm{DPPH}^{\star}$}

Se cuantificó por la medida de la disminución de la absorbancia de una solución metanólica de $\mathrm{DPPH}^{*}$ de $30 \mathrm{mg} / \mathrm{L}$ a $517 \mathrm{~nm}$ en presencia del extracto después de $30 \mathrm{~min}$ de reacción. Se expresó como capacidad antioxidante equivalente al ácido ascórbico (AEAC) usando la ecuación de Lim et al. (2007) adaptada (1):

$$
\operatorname{AEAC}(\mathrm{mg} A ́ \mathrm{~A} / 100 \mathrm{~g})=\frac{\mathrm{IC}_{50(\mathrm{AA})} \times 10^{5}}{\mathrm{IC}_{50(\text { muestra })}}
$$

Donde: $\mathrm{IC}_{50(\mathrm{~A} A)}=$ cantidad de ácido ascórbico en $1 \mathrm{~mL}$ de reacción necesaria para disminuir al 50\% la concentración inicial de $\mathrm{DPPH}^{\star}$, obtenida de graficar \% $\mathrm{DPPH}^{\star}$ remanente vs. concentración ( $\mathrm{mg} \mathrm{AA} / \mathrm{mL}$ reacción).

$\mathrm{IC}_{50 \text { (muestra) }}=$ cantidad de muestra en $1 \mathrm{~mL}$ de reacción necesaria para disminuir al $50 \%$ la concentración inicial de $\mathrm{DPPH}^{\star}$, obtenida de graficar \% $\mathrm{DPPH}^{\star}$ remanente vs. concentración ( $\mathrm{mL}$ extracto/mL reacción).

$\% \mathrm{DPPH}_{\text {remanente }}=\left[\mathrm{A}_{\text {muestra }} / \mathrm{A}_{\text {control }}\right] \times 100$

Donde:

$A_{\text {muestra }}=$ absorbancia final de la reacción entre la solución de $\mathrm{DPPH}^{\star}$ y el extracto de la muestra (materia prima, manzana mínimamente procesada control o tratada, según corresponda).

$\mathrm{A}_{\text {control }}=$ absorbancia solución de $\mathrm{DPPH}^{*}$

\section{Capacidad antioxidante por el método de FRAP (ferric reducing-antioxidant power)}

Se realizó una modificación del ensayo de FRAP propuesto por Benzie y Strain (1996). El reactivo FRAP se preparó con un buffer acetato $300 \mathrm{mM}$ (pH 3,6), una solución de $10 \mathrm{mM}$ de TPTZ en $\mathrm{HCl} 40 \mathrm{mM}$ y una solución de cloruro de hierro (III) $20 \mathrm{mM}$, en una proporción de 10:1:1 (v/v), respectivamente. $50 \mu \mathrm{L}$ de extracto se incubaron junto con $900 \mu \mathrm{L}$ de reactivo de FRAP y $130 \mu \mathrm{L}$ de agua durante 30 minutos a $37^{\circ} \mathrm{C}$. Al cabo de este tiempo se midió la absorbancia a $593 \mathrm{~nm}$. Se utilizó una curva de calibración de sulfato de hierro (II) y los resultados se expresaron en $\mu$ Moles de $\mathrm{Fe} / 100 \mathrm{~g}$ de pulpa de fruta.

\section{Ácido ascórbico y vitamina C}

La determinación de ácido ascórbico y vitamina C se realizó de acuerdo a una adaptación del método propuesto por Van de Velde et al. (2012).

\section{Extracción y determinación}

Se tomaron $25 \mathrm{~g}$ de muestra, se le agregaron $50 \mathrm{~mL}$ de solución extractante de ácido metafosfórico $(30 \mathrm{~g} / \mathrm{L})$ y ácido acético $(80 \mathrm{~g} / \mathrm{L})$ y se homogeneizó durante 1 minuto. Posteriormente la mezcla se sonicó en baño con ultrasonido y se centrifugó a 12000 g durante 20 minutos a $4{ }^{\circ} \mathrm{C}$.

Para la determinación del ácido ascórbico (ÁA) se tomó $1 \mathrm{~mL}$ del sobrenadante, se le agregó $1 \mathrm{~mL}$ de fase móvil y se filtró en membrana Millipore de $0,45 \mu \mathrm{M}$ y se inyectó en el HPLC. Para la determinación de la vitamina C (ÁAT) se tomaron $2 \mathrm{~mL}$ del sobrenadante y se le agregó $0,5 \mathrm{~mL}$ de solución de DL-ditiotreitol (DTT) $5 \mathrm{~g} / \mathrm{L}$, preparada en fosfato de potasio dibásico 2,58 M; la mezcla se dejó reaccionar durante 2 horas en la oscuridad. Luego, se tomó $1 \mathrm{~mL}$ de la mezcla de sobrenadante y solución de DTT y se le agregó $1 \mathrm{~mL}$ de fase móvil, se filtró en membrana Millipore de $0,45 \mu \mathrm{M}$ y se inyectó en el HPLC.

El contenido de ácido dehidroascórbico (ÁDHA) de las muestras se calculó como la diferencia entre los contenidos de ÁAT y ÁA (Sánchez-Mata, et al., 2000). Todas las muestras se protegieron de la luz y se manipularon en baño de hielo para evitar pérdidas por oxidación.

\section{Equipamiento \\ y condiciones cromatográficas}

Se utilizó un equipo de cromatografía líquida KONIK KNK500-A series, unido a un detector UV de longitud de onda variable (UVIS 200 Konik Instruments, Barcelona, España). Las muestras se inyectaron en el sistema cromatográfico a través de un inyector manual Rheodyne $7725 \mathrm{i}$ provisto de un loop de $20 \mu \mathrm{L}$ (Rheodyne, Berkeley, CA, EE.UU.).

La fase móvil consistió de 95\% buffer acetato de sodio/ ácido acético 0,03 M y 5\% metanol. El pH se ajustó a 5,8 y la fase móvil fue filtrada a través de una membrana Millipore de $0,45 \mu \mathrm{M}$ y desgasificada usando vacío.

La separación se realizó en una columna de fase reversa Phenomenex Gemini $5 \mu$ C18 110A con un guardacolumna (Phenomenez Inc. CA, USA) a temperatura ambiente $\left(25^{\circ} \mathrm{C}\right)$. La velocidad de flujo se fijó en $1,15 \mathrm{~mL} / \mathrm{min}$ y las medidas se realizaron a $251 \mathrm{~nm}$.

\section{Análisis microbiológicos}

$10 \mathrm{~g}$ de muestra se trituraron en $90 \mathrm{~mL}$ en agua de peptona al $0,1 \%$ en stomacher. Las diluciones decimales necesarias se sembraron en los distintos medios de cultivo por duplicado. Las placas se incubaron durante los tiempos y a las temperaturas específicas para cada recuento. Los resultados se expresaron en log UFC/g.

\section{Aerobios mesófilos totales (AMT)}

Se empleó Agar para Recuento en Placa y se incubaron a $30{ }^{\circ} \mathrm{C}$ durante 48 horas. 


\section{Psicrotrofos}

Se empleó Agar para Recuento en Placa. Las placas se incubaron a $7^{\circ} \mathrm{C}$ durante 10 días.

\section{Mohos y levaduras}

Se empleó Agar Extracto de levadura, glucosa y cloranfenicol. Las placas se incubaron a $28^{\circ} \mathrm{C}$ durante 3 a 5 días.

\section{Evaluación sensorial}

Un panel sensorial entrenado del Instituto de Tecnología de Alimentos (ITA-FIQ-UNL), compuesto por ocho jueces con experiencia en evaluación de vegetales mínimamente procesados, evaluó todas las muestras. Los atributos estudiados, seleccionados de acuerdo a Rodríguez Arzuaga (2015), fueron: apariencia general, pardeamiento, sabor característico, gusto ácido, crujencia, astringencia, jugosidad, sabores extraños y olores extraños. Se utilizaron escalas no estructuradas de $10 \mathrm{~cm}$ con términos ancla ubicados a $1 \mathrm{~cm}$ de cada extremo: "1:Mala" y "9:Muy buena" para apariencia general, "1:Muy poco" y "9:Mucho" para pardeamiento, crujencia, jugosidad y astringencia, y "1:Apenas perceptible" y "9:Muy perceptible" para sabor característico, gusto ácido, sabores extraños y olores extraños.

\section{Análisis estadístico}

Se realizaron análisis de varianza para comparar los resultados obtenidos para los distintos tratamientos. Se aplicó el test de Tukey $(\mathrm{p}<0,05)$ para detectar diferencias significativas entre muestras. Se utilizó el software Statgraphics Centurion XV.

\section{Resultados y Discusión}

No se detectaron diferencias significativas $(p>0,05)$ en el $\mathrm{pH}$ y firmeza de las muestras MP y C (Tabla 1), por lo que el mínimo procesamiento sin tratamiento químico no afectó estos parámetros. Por otro lado, el mínimo procesamiento con tratamiento (muestra $\mathrm{T}$ ) redujo significativamente $(\mathrm{p}<0,05)$ el $\mathrm{pH}$ respecto a MP, lo que era esperable por la aplicación de una solución con $\mathrm{pH} 2,81$. En cuanto a la firmeza, no se detectó diferencia significativa entre las muestras $\mathrm{C}$ y T $(\mathrm{p}>0,05)$, sin embargo la firmeza de la muestra tratada químicamente (T) fue $10,8 \%$ menor que la materia prima (MP). El mínimo procesamiento de las manzanas puede contribuir a la pérdida de firmeza debido a la acción de enzimas pécticas. Al cortar las superficies, la compartimentación subcelular se rompe y se ponen en contacto enzimas y sustratos que en el tejido intacto generalmente están separados, pudiendo comenzar reacciones que en condiciones normales no ocurren (Rojas-Graü, et al., 2008). Por lo tanto, de acuerdo a los resultados obtenidos en este estudio la pérdida de firmeza se atribuiría a las operaciones físicas asociadas al mínimo procesamiento y no a la aplicación del tratamiento químico.

La materia prima (MP) presentó el mayor contenido de sólidos solubles, seguida por la muestra T. Esto puede explicarse por la lixiviación de compuestos solubles al sumergir las manzanas en las soluciones. En el caso de T, la reducción del contenido de sólidos solubles es menor que en la muestra control (C) (Tabla 1) porque la solución aplicada tiene un contenido de sólidos solubles de $2,3^{\circ}$ Brix.

\begin{tabular}{|c|c|c|c|}
\hline Muestra & $\mathrm{pH}$ & Sólidos solubles $\left({ }^{\circ}\right.$ Brix $)$ & Firmeza $(\mathrm{N})$ \\
\hline MP & $3,44^{\mathrm{b}}$ & $11,8^{\mathrm{c}}$ & $68,6^{\mathrm{b}}$ \\
$\mathrm{C}$ & $3,43^{\mathrm{a}, \mathrm{b}}$ & $10,2^{\mathrm{a}}$ & $65,6^{\mathrm{a}, \mathrm{b}}$ \\
$\mathrm{T}$ & $3,35^{\mathrm{a}}$ & $11,0^{\mathrm{b}}$ & $61,2^{\mathrm{a}}$ \\
\hline
\end{tabular}

Letras distintas entre filas indican diferencia significativa $(\mathrm{p}<0,05$, test de Tukey). MP: materia prima; C: control; T: tratada.

Tabla 1. pH, sólidos solubles y firmeza de las muestras MP, C y T de manzanas Granny Smith.

Otros autores han reportado valores de sólidos solubles, $\mathrm{pH}$ y firmeza de manzanas Granny Smith similares a los aquí determinados para la materia prima (Gómez, et al., 2012; Altisent, et al., 2014). Rodríguez Arzuaga et al. (2013a) compararon el comportamiento de manzanas Granny Smith sin mínimo procesamiento (MP), mínimamente procesadas sin tratamiento químico (T1) y tratadas químicamente con [0,5\% ÁC+0,5\% ÁA+0,25\% cloruro de calcio] (T2) y con $[1,0 \%$ ÁC+1,0\% ÁA+0,5\% cloruro de calcio] (T3). Para la materia prima reportaron resultados muy similares a los aquí presentados (pH 3,43; sólidos solubles 12,3; ${ }^{\circ}$ Brix y firmeza $71,36 \mathrm{~N}$ ) y también observaron que al procesar la muestra sin tratamiento químico el contenido de sólidos solubles descendió y el pH se mantuvo, pero obtuvieron una disminución en la firmeza. A su vez, los tratamientos químicos aplicados por los autores generaron un descenso del $\mathrm{pH}$, la firmeza y los sólidos solubles respecto a la materia prima, que es el mismo comportamiento observado en este estudio.

En la Tabla 2 se presentan los resultados de los parámetros de color $L^{\star}, a^{*}, b^{*}, C_{a b}{ }^{*} y h_{a b}$. $L^{\star}$ representa la luminosidad, mayores valores de $\mathrm{L}^{\star}$ corresponden a muestras más claras, mientras que valores negativos de $\mathrm{a}^{\star}$ se corresponden con tonos verdes y valores positivos con tonos rojos. Las manzanas tratadas $(\mathrm{T})$ presentaron los mayores valores de luminosidad $\left(\mathrm{L}^{*}\right)$ y ángulo de tono $\left(\mathrm{h}_{\mathrm{ab}}\right)$ y el menor valor de $\mathrm{a}^{*}$. El pardeamiento enzimático ocurre a través de la oxidación de orto-fenoles a quinonas, por la acción de sistemas enzimáticos tales como la polifenol oxidasa (PPO), que luego se polimerizan a pigmentos amarronados. La PPO generalmente se asocia al plastidio y a los sustratos fenólicos que se encuentran en la vacuola, pero la disrupción celular y extracelular, que ocurre en las etapas de pelado, descorazonado y corte del mínimo procesamiento, permite que el sustrato y la enzima se pongan en contacto y, por lo tanto, reaccionen para producir pardeamiento (Roksana, et al., 2013). El resultado de la reacción de pardeamiento se ve reflejado en una disminución de $L^{*}$ (muestra más oscura) y un aumento de $\mathrm{a}^{\star}$ (pérdida de tonos verdes o desarrollo de tonos rojizos), por ende, el hecho de que la muestra $\mathrm{T}$ presente la luminosidad más alta y el $\mathrm{a}^{\star}$ más bajo puede atribuirse a que el tratamiento químico aplicado inhibió o retardó el pardeamiento. Las manzanas $\mathrm{T}$ también presentaron los valores de $\mathrm{b}^{*} \mathrm{y} \mathrm{C}_{\mathrm{ab}}{ }^{*}$ más bajos (color menos intenso), aunque para estos parámetros no se detectó diferencia significativa $(\mathrm{p}>0,05)$ entre $\mathrm{T}$ y $\mathrm{C}$. Por otro lado, no se detectaron diferencias significativas entre $\mathrm{MP}$ y $\mathrm{C}$ para ninguno de los parámetros instrumentales de color determinados.

Altisent et al. (2014) midieron el color de manzanas Granny Smith reportando valores de $L^{*}$ de 79,6 y de $h_{a b}$ de 111,3 que son comparables con los datos obtenidos en este estudio para MP. Piagentini et al. (2012) determinaron el color de 
manzanas Granny Smith mínimamente procesadas tratadas con $\left[1 \%\right.$ ÁC+1\% ÁA] y sin tratar, obteniendo valores de $\mathrm{L}^{\star}$ de alrededor de 75 y 80 , y de $a^{*}$ de 2 y $-0,8$ en las manzanas sin tratar $y$ tratadas, respectivamente. Los resultados de $\mathrm{L}^{\star}$ para ambas muestras son comparables a los aquí presentados para C y T, mientras que los de $\mathrm{a}^{\star}$ son más altos, especialmente en el caso de las manzanas sin tratamiento químico.

En su estudio, Rodríguez Arzuaga et al. (2013a) aplicaron un tratamiento químico con concentraciones similares de ácidos cítrico y ascórbico pero sin yerba mate (T3: 1,0\% ÁC $+1,0 \%$ ÁA $+0,5 \%$ cloruro de calcio) a manzanas Granny Smith mínimamente procesadas. Para las manzanas tratadas reportaron valores $\mathrm{de}^{*}{ }^{\star}$ significativamente menores $\mathrm{y}$ valores de $h_{a b}$ significativamente mayores que para las manzanas mínimamente procesadas sin tratar, coincidiendo con los valores presentados en la Tabla 2. Sin embargo, estos autores no detectaron diferencias significativas entre los valores de $\mathrm{L}^{\star}$ de ambas muestras. Estos resultados podrían sugerir que el tratamiento químico con YM aquí propuesto es más eficiente para evitar el oscurecimiento de las manzanas que uno similar sin YM.

\begin{tabular}{|c|c|c|c|c|c|}
\hline Muestra & $\mathbf{L}^{*}$ & $\mathbf{a}^{*}$ & $\mathbf{b}^{*}$ & $\mathbf{C}_{\mathrm{ab}^{*}}$ & $\mathbf{h}_{\mathrm{ab}}$ \\
\hline MP & $76,40^{\mathrm{a}}$ & $-0,38^{\mathrm{b}}$ & $17,66^{\mathrm{b}}$ & $17,68^{\mathrm{b}}$ & $91,25^{\mathrm{a}}$ \\
C & $75,87^{\mathrm{a}}$ & $-0,22^{\mathrm{b}}$ & $16,71^{\mathrm{a}, \mathrm{b}}$ & $16,71^{\mathrm{a}, \mathrm{b}}$ & $90,80^{\mathrm{a}}$ \\
T & $77,77^{\mathrm{b}}$ & $-1,31^{\mathrm{a}}$ & $16,27^{\mathrm{a}}$ & $16,33^{\mathrm{a}}$ & $94,75^{\mathrm{c}}$ \\
\hline
\end{tabular}

Letras distintas entre filas indican diferencia significativa $(\mathrm{p}<0,05$, test de Tukey). MP: materia prima; C: control; T: tratada.

Tabla 2. Parámetros instrumentales de color de las muestras MP, C y T de manzanas Granny Smith.

Los resultados de polifenoles totales se expresan en el Gráfico 1. La YM presente en la solución aplicada a las muestras $\mathrm{T}$ es rica en compuestos polifenólicos (Bravo et al., 2007), lo que se traduce en un incremento de estos compuestos en las manzanas tratadas ( $40 \%$ superior a MP). Por otro lado, C presenta un contenido significativamente menor de polifenoles que MP, posiblemente por la pérdida durante el lavado con agua (Gráfico 1). Similarmente, Rodríguez Arzuaga et al. (2013a) obtuvieron el menor contenido de polifenoles en las manzanas mínimamente procesadas lavadas con agua. Altisent et al. (2014) reportaron un contenido de polifenoles totales de Granny Smith de 54,8 mg $\mathrm{AGE} / 100 \mathrm{~g}$, que es comparable con el resultado obtenido en este estudio para MP (61,3 mg ÁGE/100 g).

En el Gráfico 2 se presentan los resultados correspondientes al ácido ascórbico (ÁA) y ácido ascórbico total (ÁAT) o vitamina C (ÁA + ácido dehidroascórbico) obtenidos para las tres muestras evaluadas. T presentó un contenido significativamente mayor $(\mathrm{p}<0,05)$ de ÁA y ÁAT, lo que demuestra que al menos parte del ácido ascórbico presente en la solución aplicada penetró las manzanas. No se detectó diferencia significativa $(p>0,05)$ entre los resultados obtenidos para las muestras MP y C, por lo que se puede concluir que el mínimo procesamiento y el lavado con agua no tienen un efecto inmediato sobre el ácido ascórbico naturalmente presente en las manzanas. Altisent et al. (2014) analizaron el contenido de ácido ascórbico y ácido ascórbico total de manzanas Granny Smith y obtuvieron 1,8 y $2,2 \mathrm{mg} / 100 \mathrm{~g}$, respectivamente. Comparando esos resultados con los aquí presentados para MP $(1,5 \mathrm{y}, 1,8 \mathrm{mg} / 100 \mathrm{~g})$, se observa que son muy similares.

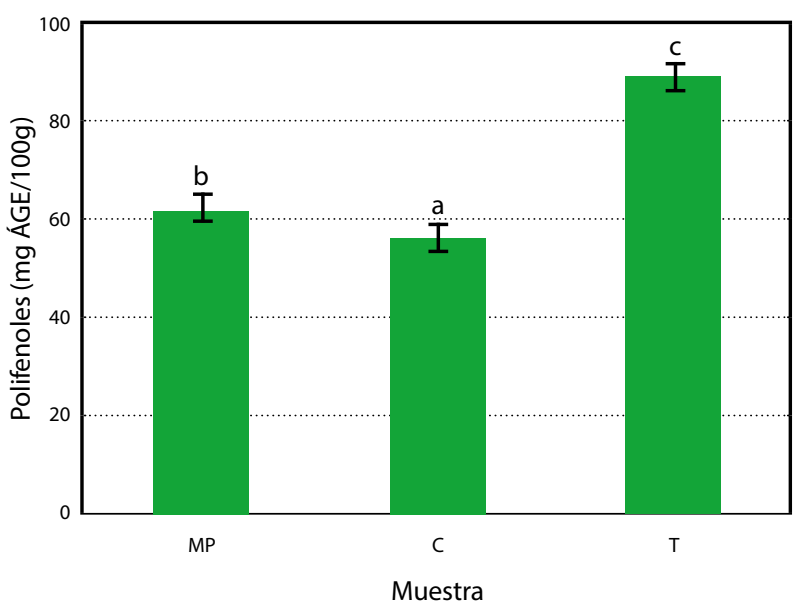

Letras distintas indican diferencia significativa ( $\mathrm{p}<0,05$, test de Tukey) entre muestras. MP: materia prima; C: control; T: tratada.

Gráfico 1. Contenido de polifenoles de las muestras MP, C y T de manzanas Granny Smith.

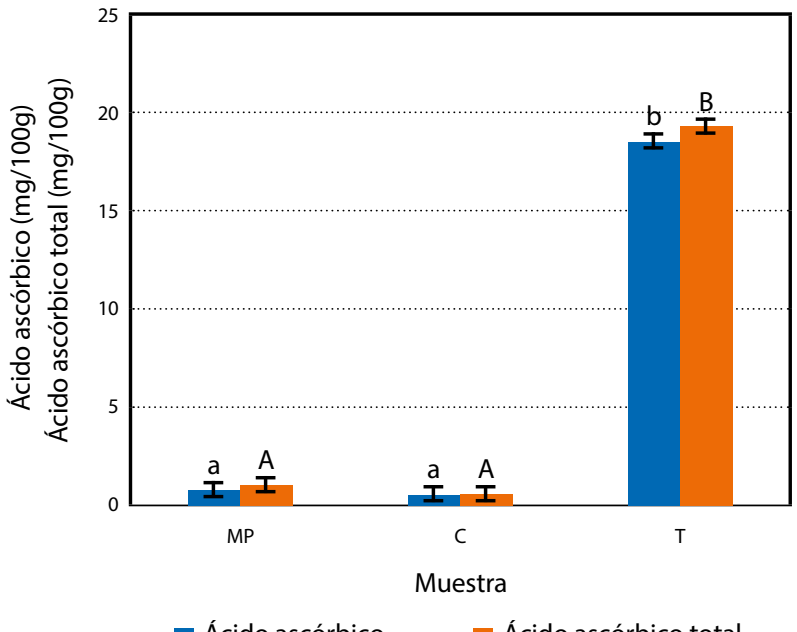

Gráfico 2. Contenido de ácido ascórbico y ácido ascórbico total de las muestras MP, C y T de manzanas Granny Smith.

MP: materia prima; C: control; T: tratada. Letras distintas dentro de cada serie de datos indican diferencia significativa ( $\mathrm{p}<0,05$, test de Tukey) entre muestras para cada parámetro

El ácido ascórbico representa el 86, 93 y 96\% del ácido ascórbico total presente en MP, C y T, respectivamente. Esto es positivo desde el punto de vista nutricional, ya que el ácido L-ascórbico es el isómero biológicamente activo con mayor actividad como vitamina $\mathrm{C}$, mientras que aún hay discusión sobre la actividad vitamínica del ácido L-dehidroascórbico (ÁDHA), aunque algunos autores indican que tiene un $10 \%$ de la actividad como vitamina C del ÁA (Nováková, et al., 2008).

En el Gráfico 3 se presentan los resultados de capacidad antioxidante (CAO) obtenidos por los dos métodos aplicados. En ambos casos, T presentó la mayor actividad antioxidante (27 y $43 \%$ superior a MP, determinadas por $\mathrm{DPPH}^{\star}$ y FRAP, respectivamente) y no se detectó diferencia significativa $(\mathrm{p}>0,05)$ entre MP y C. El aumento en la CAO en T se debe tanto al aporte de polifenoles realizado por la YM, como al ácido ascórbico presente en la solución antioxidante aplicada. 


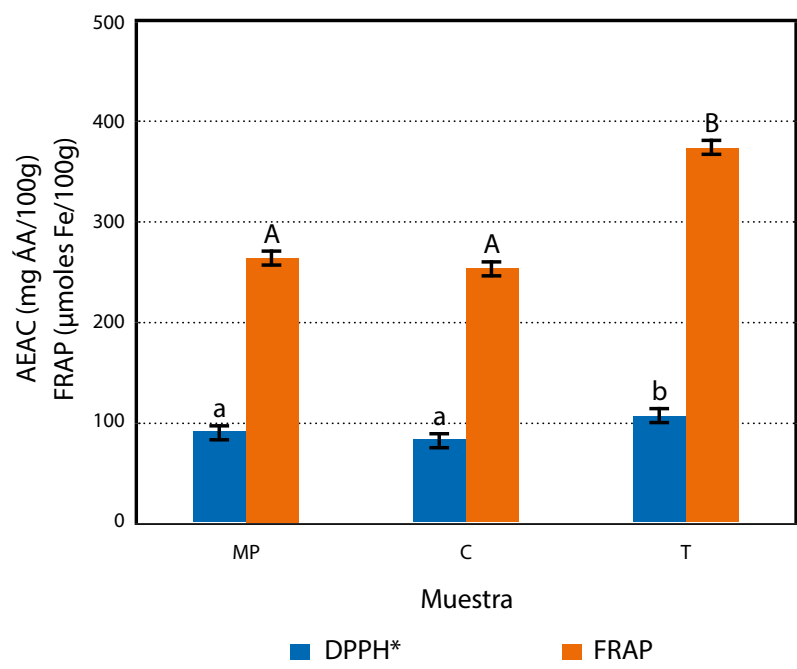

MP: materia prima; C: control; T: tratada. Letras distintas dentro de cada serie de datos indican diferencia significativa $(\mathrm{p}<0,05$, test de Tukey) entre muestras para cada parámetro.

Gráfico 3. Capacidad antioxidante por los métodos del radical $\mathrm{DPPH}^{\star}$ y FRAP, de las muestras MP, C y T de manzanas Granny Smith.

La disminución del contenido de polifenoles de C no se tradujo en un descenso significativo de su capacidad antioxidante.

En general, los recuentos microbiológicos obtenidos no superaron los 2 órdenes logarítmicos (Tabla 3). No se detectaron diferencias significativas $(\mathrm{p}>0,05)$ entre MP, C y $\mathrm{T}$ para psicrotrofos, mohos y levaduras. Estos resultados sugieren que el mínimo procesamiento, con o sin tratamiento químico, no aumentó la carga de estos microorganismos. En cuanto a los aerobios mesófilos totales (AMT), C presentó un contenido significativamente menor, mientras que no hubo diferencias significativas entre los resultados obtenidos para MP y T. Se realizaron recuentos microbiológicos de AMT, psicrotrofos, mohos y levaduras en la solución antioxidante aplicada obteniéndose recuentos inferiores a $10 \mathrm{UFC} / \mathrm{g}$. Wu et al. (2012) determinaron la carga de aerobios mesófilos totales, psicrotrofos y mohos y levaduras en manzanas Fuji frescas cortadas sin tratamiento químico y obtuvieron valores de 2,1, 2,6 y $3,1 \log$ UFC/g, respectivamente. Rojas-Graü et al. (2008) también determinaron AMT en manzanas 'Fuji' frescas cortadas sin tratar químicamente y obtuvieron cargas de 3 log UFC/g. Ambos estudios presentan recuentos algo superiores a los correspondientes a la muestra C (Tabla 3).

\begin{tabular}{|c|c|c|c|c|}
\hline Muestra & $\begin{array}{c}\text { AMT } \\
(\log \text { UFC/g) }\end{array}$ & $\begin{array}{c}\text { Psicrotrofos } \\
(\log \text { UFC/g) }\end{array}$ & $\begin{array}{c}\text { Mohos } \\
(\log \text { UFC/g) }\end{array}$ & $\begin{array}{c}\text { Levaduras } \\
(\log \text { UFC/g) })\end{array}$ \\
\hline MP & $1,6^{\mathrm{b}}$ & $1,4^{\mathrm{a}}$ & $1,4^{\mathrm{a}}$ & $1,2^{\mathrm{a}}$ \\
$\mathrm{C}$ & $1,0^{\mathrm{a}}$ & $1,0^{\mathrm{a}}$ & $1,1^{\mathrm{a}}$ & $1,0^{\mathrm{a}}$ \\
$\mathrm{T}$ & $1,7^{\mathrm{b}}$ & $1,1^{\mathrm{a}}$ & $1,0^{\mathrm{a}}$ & $1,0^{\mathrm{a}}$ \\
\hline
\end{tabular}

Letras distintas entre filas indican diferencia significativa $(p<0,05$, test de Tukey). MP: materia prima; C: control; T: tratada.

Tabla 3. Recuento de microorganismos aerobios mesófilos totales (AMT), psicrotrofos, mohos y levaduras de las muestras MP, C y T de manzanas Granny Smith.

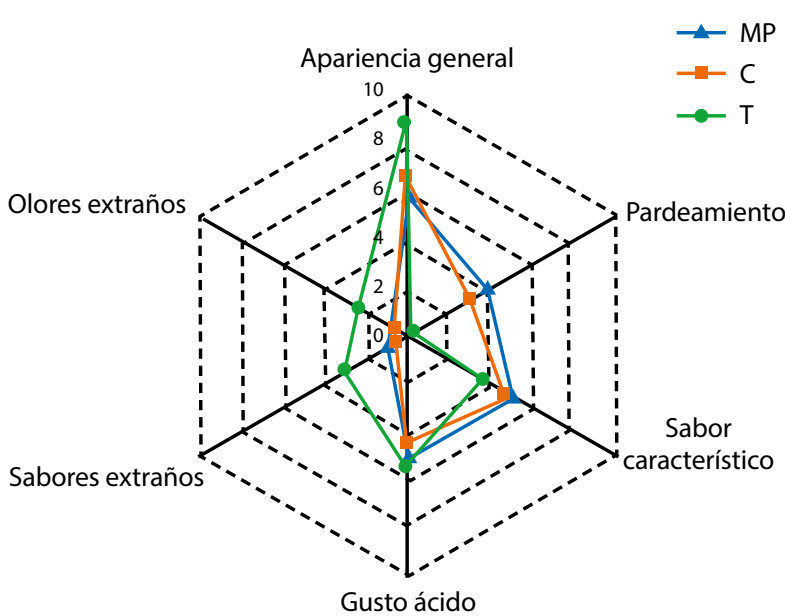

MP: materia prima; C: control; T: tratada.

Gráfico 4. Gráfico de araña para los atributos sensoriales: apariencia general, pardeamiento, sabor característico, gusto ácido, sabores extraños y olores extraños, de las muestras MP, C y T de manzanas Granny Smith.

El mínimo procesamiento y el tratamiento químico no afectaron significativamente $(\mathrm{p}>0,05)$ la astringencia, crujencia y jugosidad de las manzanas, con valores promedio $1,5 \pm 0,9 ; 8,0 \pm 0,8$ y 7,6 $\pm 0,9$, respectivamente. La materia prima (MP) obtuvo el menor valor en apariencia general $(6,1 \pm 0,7)$ y el mayor valor en pardeamiento $(4,1 \pm 1,0)$. Por otra parte, la muestra tratada $(\mathrm{T})$ obtuvo el mayor valor en apariencia general $(9,0 \pm 0,4)$ y el menor desarrollo de pardeamiento $(0,3 \pm 0,4)$ (Gráfico 4). Estos resultados indican que el tratamiento químico ejerce un efecto inmediato evitando el desarrollo de pardeamiento enzimático en las manzanas. A su vez, el hecho de que la muestra $\mathrm{C}$ presente mejor apariencia $(6,9 \pm 1,1)$ que MP puede deberse a que el lavado de los octavos de manzana reduce el contenido de sustancias que actúan como sustrato en la reacción de pardeamiento enzimático, lo que coincide con el menor contenido de polifenoles totales determinado (Gráfico 1). Las operaciones básicas de procesamiento mínimo (lavado, pelado, descorazonado y cortado) no influyeron en el sabor característico, gusto ácido y presencia de sabores y olores extraños (no se obtuvieron diferencias significativas entre MP y C) (Gráfico 4). Sin embargo, el tratamiento químico disminuyó ligeramente el sabor característico y aumentó entre 0,5 y 2,3 puntos el gusto ácido, sabores extraños y olores extraños (Gráfico 4).

Comparando los resultados instrumentales con los resultados sensoriales para cada una de las muestras, se puede observar que T presentó el menor $\mathrm{pH}$ y eso se reflejó sensorialmente, ya que fue la muestra con mayor gusto ácido. En cuanto a la firmeza, si bien instrumentalmente se detectó un descenso en las manzanas tratadas, la pérdida de firmeza no generó pérdida de crujencia de las muestras (las tres fueron similares desde el punto de vista estadístico). Los resultados instrumentales y sensoriales coincidieron en que $\mathrm{T}$ fue la muestra que desarrolló menor pardeamiento (mayor $\mathrm{L}^{\star}$, menor $\mathrm{a}^{\star}$, menor $\mathrm{C}_{\mathrm{ab}}{ }^{\star} \mathrm{y}$ menor puntaje en el atributo pardeamiento). 


\section{Conclusiones}

El mínimo procesamiento realizado sin tratamiento químico no afectó el $\mathrm{pH}$, la firmeza ni los parámetros instrumentales de color, pero sí generó un descenso significativo del contenido de sólidos solubles y del recuento de microorganismos aerobios mesófilos totales. En cuanto al potencial saludable, disminuyó el contenido de polifenoles totales, pero no el contenido de ácido ascórbico y vitamina $\mathrm{C}$ y la capacidad antioxidante. No se vieron afectadas las características sensoriales relativas al sabor (sabor característico, gusto ácido, sabores extraños y olores extraños), así como la astringencia, crujencia y jugosidad de las muestras, pero sí se obtuvo mejor apariencia general y menor desarrollo de pardeamiento en C que en MP.

La aplicación de la solución con 1,2\% YM; 0,9\% ÁC y $1,0 \%$ ÁA produjo un incremento del contenido de sólidos solubles y de los parámetros de color $\mathrm{L}^{*} \mathrm{y} \mathrm{h}_{\mathrm{ab}}$, $\mathrm{y}$ un descenso del parámetro $\mathrm{a}^{*}$, respecto de la muestra $\mathrm{C}$. Los valores de los parámetros instrumentales de color junto con la mejora de la apariencia general sensorial y del menor pardeamiento evaluado sensorialmente pone en evidencia que el tratamiento químico aplicado fue efectivo en controlar el desarrollo de pardeamiento enzimático, así como en incrementar significativamente $(p<0,05)$ el potencial saludable de manzanas Granny Smith mínimamente procesadas (mayor contenido de polifenoles y vitamina $C$, y mayor capacidad antioxidante), manteniendo bajos recuentos microbiológicos similares a los encontrados en la materia prima.

\section{Reconocimientos}

Trabajo realizado con fondos de la Universidad Nacional del Litoral a través de la programación CAI+D 2011. El Laboratorio Tecnológico del Uruguay (LATU) financió la estadía de Mariana Rodríguez Arzuaga en Santa Fe. Se agradece a la Mag. Cecilia Bernardi, a la Lic. Nora Sabbag y a la Tca. María A. Moguilevsky por su asistencia técnica en este trabajo.

\section{Referencias}

Alegre, I., Viñas, I., Usall, J., Anguera, M. y Abadias, M., 2011. Microbiological and physicochemical quality of fresh-cut Apple enriched with the probiotic strain Lactobacillus rhamnosus GG. En: Food Microbiology, 28, pp.59-66.

Altisent, R., Plaza, L., Alegre, I., Viñas, I. y Abadias, M., 2014. Comparative study of improved vs. traditional apple cultivars and their aptitude to be minimally processed as 'ready to eat' apple wedges. En: LWT-Food Science and Technology, 58, pp.541-549.

Benzie, I.y Strain,J., 1996. The ferric reducing ability plasma (FRAP) as a measure of "antioxidant power": the FRAP assay. En: Analytical Biochemistry, 239, pp.70-76.

Bravo, L., Goya L. y Lecumberri, E., 2007. LC/MS characterization of phenolic constituents of mate (Ilex paraguariensis, St. Hil.) and its antioxidant activity compared to commonly consumed beverages. En: Food Research International, 40, pp.393-405.

Cano, M.P., Sánchez-Moreno, C., de Pascual-Teresa y S., de Ancos, B.J.C., 2005. Procesado mínimo y valor nutricional. En: González-Aguilar, G.A., Gardea, A.A. y CuameaNavarro, F., ed., 2005. Nuevas tecnologías de conservación de productos vegetales frescos cortados. Hermosillo: Centro de Investigación en Alimentación y Desarrollo, pp.155-176.

Chung, H. y Moon, K., 2009. Browning characteristics of fresh-cut 'Tsugaru' apples as affected by pre-slicing storage atmospheres. En: Food Chemistry, 114, pp.1433-1437.

Gómez, P., García-Loredo, A., Nieto, A., Salvatori, D. Guerrero, S. y Alzamora, S., 2012. Effect of pulsed light combined with an antibrowning pretreatment on quality of fresh cut apple. En: Innovative Food Science \& Emerging Technologies, 16, pp.102-112.

Lanciotti, R., Gianotti, A., Patrignani, F., Belletti, N., Guerzoni, M.E. y Gardini, F., 2004. Use of natural aroma compounds to improve shelf-life and safety of minimally processed fruits. En: Trends in Food Science \& Technology, 15, pp.201-208.

Lim, Y.Y., Lim. T.T. y Tee. J.J., 2007. Antioxidant properties of several tropical fruits: a comparative study. En: Food Chemistry, 103, pp.1003-1008.

Martín, L., Bernardi, C. y Piagentini, A.M., 2010. Inhibición de la actividad de la enzima polifenoloxidasa de manzana var. Princesa con extractos de yerba mate. En: AUGM, UNL. Actas de la XVIII Jornadas de Jóvenes Investigadores, Asociación de Universidades Grupo Montevideo (AUGM). Santa Fe: AUGM, UNL.

Nováková, L., Solich, P. y Solichová, D., 2008. HPLC methods for simultaneous determination of ascorbic and dehydroascorbic acids. En: Trends in Analytical Chemistry, 27(10), pp.942-958.

Piagentini, A.M. y Güemes, D.R., 2002. Shelf life of freshcut spinach as affected by chemical treatment and type of packaging film. En: Brazilian Journal of Chemical Engineering, 19(4), pp.383-389.

Piagentini, A.M., Güemes, D.R. y Pirovani, M.E., 2002. Sensory characteristics of fresh-cut spinach preserved by combined factors methodology. En: Journal of Food Science, 67(4), pp.1544-1549.

Piagentini, A.M., Güemes, D.R. y Pirovani, M.E., 2003. Mesophilic aerobic population of fresh-cut spinach as affected by chemical treatment and type of packaging film. En: Journal of Food Science, 68(2), pp.602-607.

Piagentini, A., Martín, L., Bernardi, C., Güemes, D. y Pirovani, M., 2012. Color changes in fresh-cut fruits as affected by cultivar, chemical treatment and storage time and temperature. En: Caivano, J.L. y Buera, M.P., ed., 2012. Color in Food: Technological and Psychophysical Aspects. Boca Raton: CRC. pp.263-270.

Piagentini, A.M. y Pirovani, M.E., 2016. Total phenolics content, antioxidant capacity, physicochemical attributes and browning susceptibility of different apple cultivars for minimal processing. En:International Journal of Fruits Science, en prensa.

Pirovani, M.E., Piagentini, A.M., Güemes, D.R. y Arkwright, S., 2004. Reduction of chlorine concentration and microbial load during washing-disinfection of shredded lettuce. En: International Journal of Food Science and Technology, 39, pp.341-347.

Pirovani, M.E., Piagentini, A.M., Güemes, D.R., Rodríguez, S.C., Qüesta, A.G. y Casóliba, R.M., 2009. Calidad sensorial y nutricional de vegetales de hojas frescos cortados. En: González Aguilar, G.A., Álvarez Parrilla, E., de la Rosa, L., Olivas, I.G. y Ayala Zavala, J.F., ed., 2009. Aspectos nutricionales y sensoriales de vegetales frescos cortados. México : Trillas, CIAD-UAJC. pp.64-97. 
Pirovani, M., Piagentini, A.M. y Van De Velde, F., 2015. Bioactive compounds in fresh-cut fruits: occurrence and impact of processing and cold storage. En: Gupta, V.K., Tuohy, M.G., O'Donovan, A. y Lohani, M., ed., 2015. Biotechnology of bioactive compounds: sources and applications in foods and pharmaceuticals. London: John Wiley \& Sons. p.736.

Rico, D., Martín-Diana, A.B., Barat, J.M. y Barry-Ryan, C., 2007. Extending and measuring the quality of fresh cut fruit and vegetable: a review. En: Trends in Food Science \& Technology, 18, pp.373-386.

Rodríguez Arzuaga, M., 2015. Aplicación de tratamientos térmicos suaves $y$ antioxidantes de origen natural a manzanas frescas cortadas. Santa Fe: Universidad Nacional del Litoral. (Tesis de Maestría).

Rodríguez Arzuaga, M., Güemes, D., Benavides, M., Rivas, M., Pirovani, M. y Piagentini, A., 2013a. Evaluación preliminar del efecto del tratamiento químico sobre atributos fisicoquímicos, sensoriales y bioactivos de manzanas frescas cortadas. En: Revista Iberoamericana de Tecnología Postcosecha, 14(2), pp.223-229.

Rodríguez Arzuaga, M., Costa, S., Caballero, S., Sabbag, N. y Piagentini, A., 2013b. Determinación de las concentraciones óptimas de una solución de yerba mate para incrementar el potencial saludable y reducir el pardeamiento en manzanas frescas cortadas. En: CYTAL. Actas del IX Congreso de Ciencia y Tecnología de Alimentos (CYTAL). Rosario: CYTAL, AATA.
Rojas-Graü, M.A., Tapiam M.S. y Martín-Belloso, O., 2008. Using polysaccharide-based edible coatings to mantain quality of fresh-cut Fuji apples. En: LWT-Food Science and Technology, 41, pp.139-147.

Roksana, H., Wills, R.B.H., Pristijono, P. y Golding, J.B., 2013. Effect of nitric oxide (NO) and associated control treatments on the metabolism of fresh-cut apple slices in relation to development of surface browning. En: Postharvest Biology and Technology, 78, pp.16-23.

Sánchez-Mata, M.C., Cámara-Hurtado, M., Diez-Márquez, C. y Torija-Isasa, M.E., 2000. Comparison of high-performance liquid chormatography and spectrofluorimetry for vitamin $\mathrm{C}$ analysis of green beans (Phaseolus vulgaris L.). En: Europe Food Research and Technology, 210, pp.220-225.

Singleton, V. y Rossi, J.,1965. Colorimetry of total phenolics with phosphomolybdic-phosphotungstic acid reagents. En: American Journal of Enology and Viticulture, 16, pp.144-158.

Toivonen, P.M.A. y Brummell, D.A., 2008. Biochemical bases of appearance and texture changes in fresh-cut fruit and vegetables. En: Postharvest Biology and Technology, 28, pp.1-14.

Van de Velde, F., Pirovani, M.E., Cámara, M.S., Güemes, D.R. y Bernardi, C., 2012. Optimization and validation of a UVHPLC for vitamin C determination in strawberries (Fragaria ananassa Duch.), using experimental designs. En: Food Analytical Methods, 5, pp.1097-1104.

Wu, Z.S., Zhang, M. y Wang, S., 2012. Effects of high pressure argon treatments on the quality of fresh-cut apples at cold storage. En: Food control, 23, pp.120-127. 